\title{
A Pictorial Interaction Language for Children to Communicate with Cultural Virtual Characters
}

\author{
Birgit Endrass ${ }^{1}$, Lynne Hall ${ }^{2}$, Colette Hume ${ }^{2}$, Sarah Tazzyman ${ }^{2}$, \\ and Elisabeth André ${ }^{1}$ \\ 1 Human Centered Multimedia, Augsburg University, \\ D-86159 Augsburg, Germany \\ \{endrass, andre\}@hcm-lab.de \\ 2 University of Sunderland, \\ Sunderland, SR6 0DD, UK \\ \{lynne.hall, colette.hume, sarah.tazzyman\}@sunderland.ac.uk
}

\begin{abstract}
In this paper, we outline the creation of an engaging and intuitive pictorial language as an interaction modality to be used by school children aged 9 to 11 years to interact with virtual characters in a cultural learning environment. Interaction takes place on a touch screen tablet computer linked to a desktop computer on which the characters are displayed. To investigate the benefit of such an interaction style, we conducted an evaluation study to compare the pictorial interaction language with a menu-driven version for the same system. Results indicate that children found the pictorial interaction language more fun and more exciting than the menus, with users expressing a desire to interact for longer using the pictorial interaction language. Thus, we think the pictorial interaction language can help support the children's experiential learning, allowing them to concentrate on the content of the cultural learning scenario.
\end{abstract}

\section{Introduction}

While traditional learning systems provide conventional interaction devices such as mouse and keyboard, especially for child users intuitive interaction is important to provide an engaging experience. Menus provide bound and restricted interaction choices, possibly limiting the user's perceived freedom in their interactions. Free text input can be desirable, however, due to technical constraints such as limited support of vocabulary and grammar this is hard to realise. Further, children's keyboard skills are often not fully developed compared to adults, reducing children's abilities to express themselves. Recent paradigm shifts towards more natural user interfaces, based on either direct touch or three-dimensional spatial interaction [1] provide interesting alternatives to increasing user engagement, particularly for children. One of the most often stated benefits is the 
view that interacting with an application through directly touching graphical elements is a more "natural" or "compelling" approach than working indirectly with a mouse or other pointing devices [2].

In this paper, we investigate a game play interaction modality designed for, and with children. We present a pictorial interaction language using touch-based gestures on a tablet computer that allows children to interact freely with characters displayed on a different screen. The interaction is developed for use in playing a serious game in which children communicate with a virtual character to learn about resolving a cultural conflict.

\section{Background}

This paper focuses on the development and evaluation of a pictorial interaction language for children aged 9 to 11 years. This interaction modality is part of the eCute project [3], which aims to create and encourage cultural awareness among children.

In the MIXER showcase [4], the user plays the role of an invisible friend to provide advice and support to a virtual character, called Tom. The narrative of the MIXER application centres on Tom visiting a summer camp where he meets a group of characters that he knew before. With this group, Tom plays a game called Werewolves (see [5] for a description of the rule set). In the game, each player is assigned a role, as either a werewolf or a villager. The aim of the game is to deduce which character in the group is the werewolf, before the werewolf kills all of the villagers. Several times during the game, Tom asks for the user's advice. After playing for a while, Tom leaves the first group of children and meets a different group that he has not met before. In this group, Tom and the user are confronted with crucial changes to the rule set by which the game is played; this leads to a critical incident and a potential conflict situation.

To create a novel, engaging and effective learning experience we aim to develop an interaction modality that is both intuitive and engaging for children of the target age group. A pictorial interaction language was identified as a solution to the problem of creating a novel and universal interaction experience.

\section{Related Work}

We think that finding novel and intuitive interaction modalities for educational systems is a crucial task. Sali and colleagues [6] investigated three different dialogue approaches for game interfaces. They found that users prefer a natural language interface over interfaces that allow users to select sentences and interfaces that make use of an abstract response menu interface. However, some users had problems with the natural language interface because they found it hard to figure out what to say in a particular situation. Compared to adults, this problem may be magnified for children. We encountered similar issues in former work [7] where children interacted with a virtual learning environment using typed text input. The interaction choice for natural language input resulted in 
several problems including recognition problems for the software coupled with the difficulty and time required for children to express themselves in typed text. We think that by using a pictorial interaction language the disadvantages of text input are reduced, whilst retaining a large degree of interaction freedom.

Pictorial languages are commonly used with children in the field of augmentative and alternative communication, e.g. in communication training for autistic children [8], [9]. Widget symbols (e.g. [10]) also find their usage on websites that provide understanding and communication for people who find reading text difficult, e.g. [11]. Their potential for intuitive communication is gaining ground for non-disabled children as well. For example, a pictorial language is used on CBBC (children's television channel) in the UK to facilitate communication. We thus see great potential in using a pictorial language as an intuitive interaction modality to communicate with virtual characters in learning environments as well.

Researchers have found that in the field of human computer interaction using a visual style of expressing oneself helps to motivate children to complete creative and challenging tasks, such as telling stories [12], or learn computer programming using storytelling environments [13].

To overcome the language barrier in inter-cultural communication, Takasaki and Mori [14] describe a communicator that was developed for children of different cultural backgrounds to be able to talk to each other using pictogram communication. This was reported to be an effective and practical user interface design method with children. In a similar manner, we aim to design a pictorial language for children to enable communication with a virtual character.

\section{Design and Realisation}

With the intention of improving both engagement and user experience for 911 year olds, we use an Apple iPad as the interaction device in combination with a pictorial language as interaction modality, provided as an extension to a desktop-based system connected via Wi-Fi.

\subsection{System Setup}

Figure 1 shows an overview of the setup including a child using it. The user can observe what happens in the virtual environment on the screen of the desktop while interaction takes place on the tablet computer. In this way, information that should only be visible to the user is shown on the tablet computer, while the environment with the virtual characters is visible for everyone. This supports the impression of being an invisible friend whose actions cannot be seen by the other characters involved in the gameplay.

As the focus of the present study was to test the suitability of the pictorial interaction language, it was tested with an early version of the MIXER game holding a virtual friend character that is involved in a fictive game with a group of characters (running in the AAA application [15]). During the game, the friend 
character asks the user for advice several times. In each case, the character leaves the group, comes closer to the screen and updates the user on what happened in the game. Depending on the context of the question, different icons are provided on the tablet computer to construct the answer message in a pre-structured "grammar", by e.g. combining an action and an emotion. After hitting the sendbutton, the friend character reacts to the message and returns back to the group of other characters.

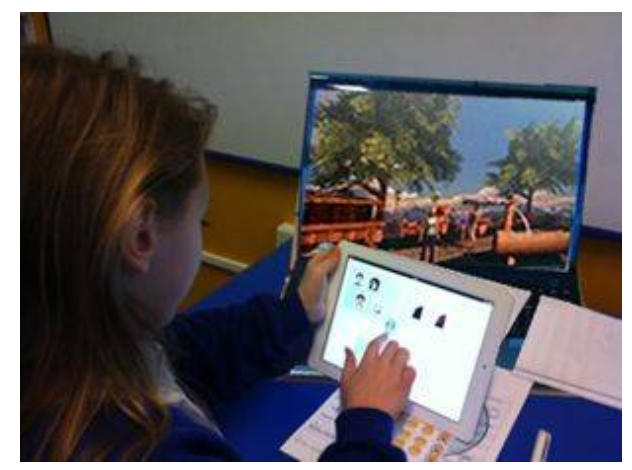

Fig. 1. Example setup, with a child using the pictorial interaction interface on an iPad

\subsection{Interaction Modes}

We designed two different advisory modes for interaction with the virtual friend character in the MIXER game:

- "During game advisory mode" to support the friend character during gameplay;

- "Critical incident advisory mode" to deal with the critical incident after playing with a different group of characters that play the same game with different rules.

In this paper, only the advisory modes that occur during the game were investigated. Therefore, we identified four different advisory modes that describe standard situations for the Werewolf game: (1) Questioning who the werewolf is, (2) Reasoning why somebody is the werewolf, (3) Reacting if somebody else is being accused, (4) Reacting if oneself is being accused.

Depending on the context of the game, the advisory modes can either be used alone or combined to simulate a longer conversation between the user and the friend character. For example, after a character has been accused of being a werewolf (3) the friend character could ask who else could be a candidate (1) and why the user thinks so (2). Interaction is managed in a question and answer style, with the friend character asking for advice and the user answering by constructing a message. 


\subsection{Vocabulary Selection}

We had to create a language that would fit our purpose of communicating with a virtual character that was playing a game of werewolves with other virtual characters. As this was a very specific requirement, we could not, for example, acquire a set of validated open source icons. It was necessary to create and test our own icon set. The first stage in the creation process was to investigate the language used while playing the werewolve game. Taking into account the rules of the game, some words were obvious, such as 'You', 'They', 'Accuse', 'Defend', 'Werewolf' etc. We recruited a total of 70 children (aged 9 to 11) who played the real world werewolf card game in small groups. The games were recorded and transcribed. In total, we identified 60 frequently used words, such as "I accused her because she looks suspicious" or "he's being too quiet". These words were later grouped, e.g. emotions or actions and structured in a way to match the identified interaction modes.

\subsection{Icon Design}

Besides following the design standards of ISO/IEC 11581 by using a consistent size, icon behaviour, and a similar design for all of our icons, a challenge was to design the icons to be sufficiently intuitive for children to construct meaningful messages.

In total, a set of over 60 icons was required for the pictorial interaction language based on the study mentioned above. The majority of the icons show a small green character. This character was shown in different positions to convey the different action states that were identified. For example, for the word 'calmly' the character was shown in a meditative pose. The colours green and red were used in the icons to convey positive and negative respectively. The icons were designed intuitively, by using what seemed to be the most appropriate visual representation for children of each word. However, what is obvious to a team of researchers is clearly not always going to be obvious to a child. Thus, we conducted a small study with 30 children to test their comprehension of the icons. We began by introducing and playing a short game of werewolves, which gave the children a context in which to discuss the meaning of the icons. The children were given activity sheets with pictures of the icons, and then asked to think about the game they had played and to try and work out what each of the icons meant. Following the game we held small focus group activities during which the children were shown the icons again and asked to discuss what the icons represented. This gave the research team useful qualitative information about children's views of the icons and their design. The icons that were not easily understood by the children became part of the next activity in which the children themselves helped to redesign the icons. These were used to develop the final icon set. The focus group activity was repeated with the final icon set with a further 25 children at a different school, during which all icons were successfully identified by the children. Figure 2(left) shows a small subset of the icons designed for our pictorial interaction language along with their intended meanings. 

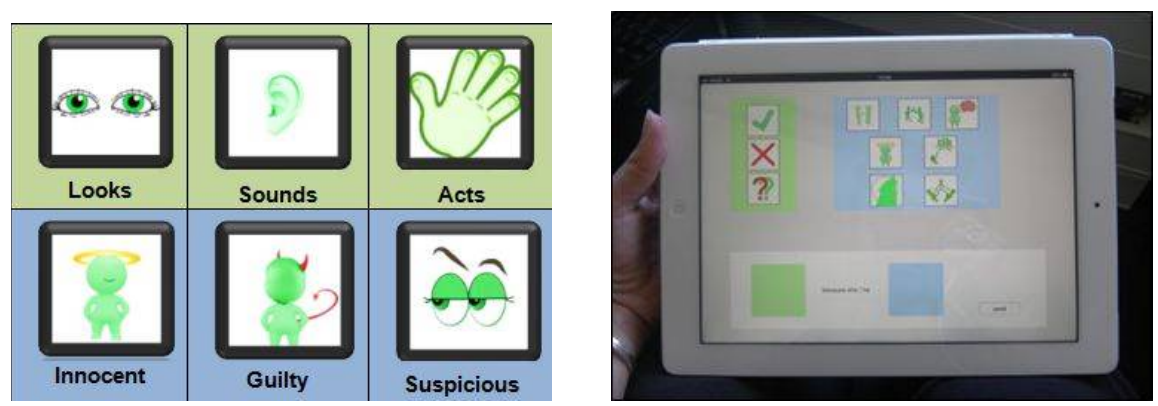

Fig. 2. Left: Example icons with intended meanings; Right: Interaction interface on the iPad

\subsection{Interface Design}

For the interface shown on the iPad, groups of icons are provided, while one icon of each group can be selected to form a sentence in a pre-structured grammar, e.g. by combining an action with an emotion. Figure 2 (right) shows the iPad with an interactive screen of the third advisory mode. Different coloured views contain the different groups of icons. Icons are moved by touching and dragging them across the screen. The white area on the lower part of the screen holds the message that the user constructs, providing empty views of the same colour of the group of icons that can be selected. The simple colour code helps the user understand that an icon of each provided group should be selected and moved to the corresponding area. Additionally, icons are automatically attached to the correct position (centre of same coloured area) as soon as they are moved into the user sentence area. In case an icon was selected for that area before it is replaced and the former is popping back to its initial position. Thus, only well formed sentences can be produced by the child, not allowing grammatically incorrect or nonsense sentences that would be uninterpretable for the system.

An example of a standard situation in the Werewolf game includes questioning why another player might be a werewolf (2). To answer this question, an action and a reason can be combined by the user. To help the user understand what kind of answers can be created, the message is initialised by the words "because he / she", followed by two different coloured views relating to actions and reasons respectively. Using the icons shown in Figure 2 (left), messages such as "because he/she looks guilty" or "because he/she acts suspicious" could, for example, be constructed.

\section{$5 \quad$ User Study}

To test the possible benefit of a pictorial interaction language over traditional interaction modalities, a user study was conducted with 9-11 year olds. 


\subsection{Interaction Modes}

For the present study, we implemented two interactive versions of our system both using the touch-based interaction on the iPad: icon-based vs. menu-based. The icon-based version contains the pictorial language described above. The menu-based version was implemented to provide a set of choices in text form, representing choices that could also be constructed with the pictorial interaction language, which can be selected by the user by clicking on them (see Figure 3 for comparison of the two iPad interfaces).

The setup of the game is constant for both versions. In each case the friend character repeats the choice of the user, comments on it and returns to the group. However, the characters' comments are limited to the number of choices in the menu-based version to ensure that users are not influenced by the wider variety of the system in the icon-based version.
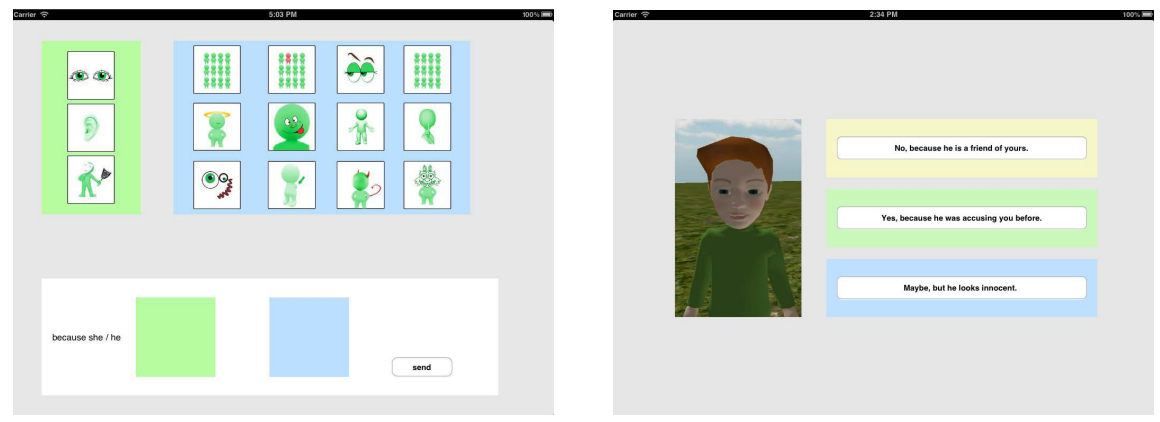

Fig. 3. Screenshots of the iPad showing icon-based interaction (left) and menu-based interaction (right)

\subsection{Expectations}

With the pictorial interaction language, we provide interpretational freedom to the users by offering many opportunities to construct sentences. In addition we want to inspire children's curiosity and exploration. For our study, we hypothesized that an icon-based interaction style would be perceived as more engaging and interesting compared to a traditional menu-based interaction.

However, a possible advantage of the menu-based version might be that it is more intuitive to use for inexperienced users, since fewer, clearer choices are provided and there is no need for children to construct their own sentences.

\subsection{Procedure}

To investigate which interaction modality the children preferred, an evaluation study was conducted with the target age group with each child having a PC, iPad and headphones. Children were introduced to the study activities, before playing both versions of the system. After using each of the versions, the children 
completed a questionnaire, then used the other version and completed the same questionnaire again.

For evaluation, a 4-part questionnaire was developed:

Part 1 provided requested descriptive data, e.g. the children's age, gender and previous exposure to tablets.

Part 2 included questions focused at gaining the child's response to their first interactive experience. Each question was provided as bi-polar adjectives using a 5-point facial scale, see Figure 4. Facial scales have been shown to be well suited for evaluation with children in school environments,see [16]. The questions included:

- Ease of use: was the application easy to use or not, and could the child achieve what they intended with the interaction

- Engagement: was the experience fun, was it exciting, would they want to play again, would they have liked to play longer

- Visual appeal and interaction comprehension: did children like the appearance of the interface and could they understand the meanings provided in the interaction dialogue (e.g. the menu items or the icons)

- Open questions asking what children liked most and least about the game

Part 3 repeated the questions in Part 2 for the second interaction experience.

Part 4 asked the child to compare the two interaction modalities and decide which had been more fun, exciting and interesting.

Finally children were given a gold star sticker and were asked to put the sticker on a picture of the version they liked the best. The gold star sticker was chosen as children recognise stars and stickers as tokens that are awarded for something that is very good i.e. stars and stickers are often given in class for a good piece of work.

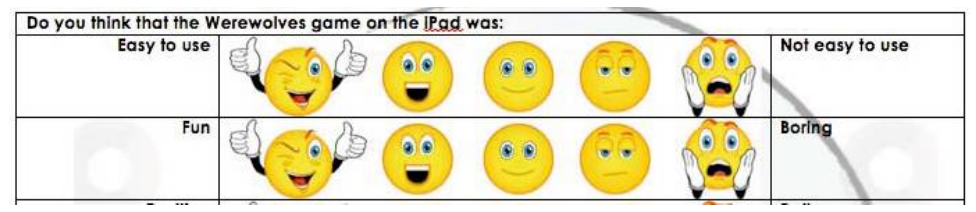

Fig. 4. Example questions from the questionnaire

71 children aged $9-11$ years $(\mathrm{M}=9.65, \mathrm{SD}: .56)$ living in the UK participated in the study. $59.2 \%(\mathrm{n}=42)$ of the sample was boys, and $40.8 \%$ girls $(\mathrm{n}=29)$. Most children had used an iPad before $(84.5 \%, \mathrm{n}=60)$.

35 of the children used the icon-based application first, followed by the menubased version, while the remaining 36 children played the versions ordered the other way round (i.e. the procedure was counterbalanced to avoid order or practice effects). 


\section{$6 \quad$ Results}

Mean values of the children's ratings are summarized in Figure 5. It shows that overall the icon-based version was rated more positively compared to the menubased version. There was one exception to this for ratings of the pictures on the iPad being easy to understand / hard to understand. For this question, children rated the menu-based interaction slightly more favourably (i.e. the menus were easier to understand).

Figure 5 also clearly illustrates that children rated all the questions positively for both the menu and icon-based interaction, with no mean ratings above 3 (scale ranged from 1 to 5 , with 1 being most favourable and 5 the least favourable). The highest (i.e. least favourable) mean response of 2.61 was for ratings of how exciting / dull the menu-based interaction was.

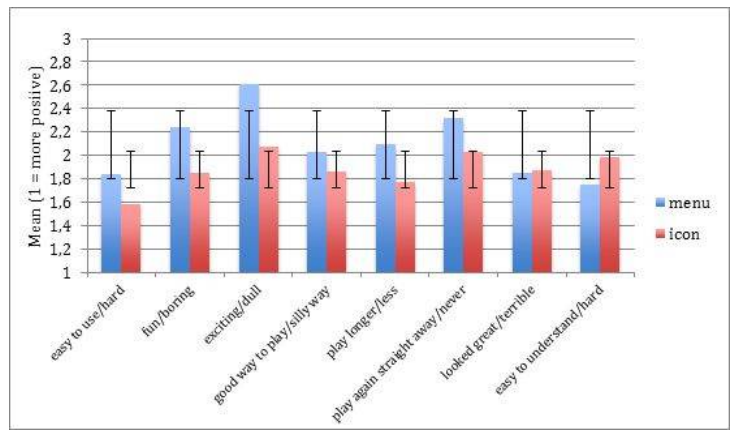

Fig. 5. Mean ratings (error bars $1 \mathrm{SD}$ ) of icon-based vs. menu-based interaction

The facial scale that children rated could not be assumed to follow an interval scale, but rather ordinal. Therefore, non-parametric Wilcoxon signed-rank tests for related samples were calculated to determine whether there were any significant differences in mean ranks between children's ratings of the menu-based versus icon-based interactions. Figure 6 illustrates the $\mathrm{Z}$ statistic, associated

\begin{tabular}{lccc}
\hline Question & $\boldsymbol{Z}$ value & $\begin{array}{c}\boldsymbol{p} \\
\text { (1-tailed) }\end{array}$ & $\boldsymbol{r}$ \\
\hline Easy to use/not easy to use & -1.92 & .03 & .23 \\
Fun/Boring & -3.10 & .001 & .37 \\
Exciting/Dull & -3.66 & $<.001$ & .43 \\
Good way to play/silly way & -1.07 & .14 & .13 \\
Play longer/less time & -2.31 & .01 & .27 \\
Play again straight away/not & 2.14 & .02 & .25 \\
at all & & & \\
looked great/terrible & -.03 & .49 & .00 \\
easy to understand /hard to & -1.52 & .06 & .18 \\
understand & & & \\
\hline
\end{tabular}

Fig. 6. Interaction questions for the menu-based and icon-based interaction, associated $\mathrm{Z}$ values, significance (1-tailed), and effect size (r) 
significance for one-tailed tests, and effect sizes (r) for each of the questions in our questionnaire.

Children rated the icon-based version (median $=1$ ) as slightly easier to use then the menu-based interaction (median $=1$ ). The icon-based interaction (median $=2$ ) was also rated as more fun to use compared to the menu-based interaction (median $=2$ ). Children rated that the icon-based interaction (median = 2 ) was more exciting compared to the menu-based interaction (median $=3$ ) and wanted to play longer with the icon-based (median $=1$ ) interaction compared to the menu-based (median $=2$ ) interaction. The icon-based interaction (median $=2$ ) was rated more favourably by children for wanting to play again straight away compared to the menu-based interaction ( median $=2$ ).

No significant differences were found between the icon (median $=2$ ) and menu-based (median $=2$ ) interactions for ratings of whether it was a good way to play the game. Children rated the design of the interface (looked great/looked terrible) on both the menu-based (median = 2) and icon-based interaction (median $=2$ ) favourably, with no significant differences reported between the two interactions. Children also found the interface for both the menu-based (median $=1$ ) and icon-based (median $=1$ ) version easy to understand, with no significant differences. Figure 7 illustrates the positive (icon $i$ menu) and negative (icon i menu) ranks derived from the Wilcoxin tests for each of the eight questions that children rated using the facial scale. The figure clearly demonstrates that children favoured the icon-based interaction over the menu-based interaction for all questions, with the exception of whether the interaction interface on the iPad was easy/hard to understand.

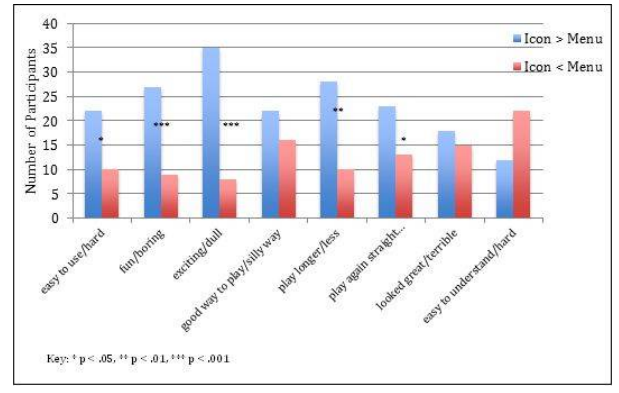

Fig. 7. Number of participants who rated the icon-based interaction more favourably than the menu-based interaction (icon \& menu), and the menu-based interaction more favourably than the icon-based interaction (icon $;$ menu)

Binomial tests (0.50) were carried out to determine whether children found the icon-based or menu-based interaction more fun, exciting and interesting. Children reported finding the icon version more fun compared to the menu version [fun $(\mathrm{Z}=5.93, \mathrm{p} ; .001)$, menus $\mathrm{n}=10(.14)$, icons $\mathrm{n}=61(.86)]$, and the icon version more exciting [exciting $(\mathrm{Z}=-2.85, \mathrm{p} ; .01)$, menus $\mathrm{n}=23(.32)$, icons $\mathrm{n}=48(.68)]$. No preference for menus or icons was reported by children 
for levels of interest [interesting $(\mathrm{Z}=.000 \mathrm{p}=1.0)$, menus $\mathrm{n}=36(.51)$, icons $\mathrm{n}$ $=35(.49)$ ].

Each child was given one sticker and asked to place this on his/her favourite version of the interaction interface. $92 \%(\mathrm{n}=55)$ of children placed their sticker on the icon-based version, leaving just $8 \%(\mathrm{n}=5)$ of children who placed it on the menu-based version. A one-sample binomial test revealed that significantly more children said that the icon-based version was their favourite compared to the menu-based version [favourite $(\mathrm{Z}=6.33$, $\mathrm{p}$ i.001), words $\mathrm{n}=5$ (.08), pictures $\mathrm{n}=55(.92)]$.

\section{Conclusions and Future Work}

This paper discusses the development and evaluation of a pictorial interaction language to test the suitability of such an interaction modality for 9-11 year old children for a cultural learning scenario. We compared the experience of the pictorial interaction language with a more traditional menu-driven interaction. Through using the same application with the same interaction device we have established that children preferred the pictorial interaction, considering it to be more fun and exciting than a menu-driven approach. In line with our expectations, the children rated the pictorial interaction as harder to understand compared to the menu-driven approach but at the same time more fun. We thus think that the pictorial language provides a more challenging interaction that positively influences the overall user experience. Our focus was to investigate the potential for a pictorial interaction approach to engage children in a games-based learning experience. Through directly comparing pictorial interaction and menu-driven interfaces, even though children were positive about both approaches, results indicate that:

- Children found the pictorial interaction to be more fun than the menu-driven version.

- Pictorial interaction was perceived as more exciting than menus

- Children would have liked to play longer with the pictorial interaction than with the menu-driven system

- Pictorial interaction is well suited for, and preferred by, the age group with just $8 \%$ of the children preferring the menu-driven version.

The pictorial interaction language is now integrated into the complete cultural conflict learning experience. Studies conducted in Germany and UK with the full system will further establish the benefits of an intuitive pictorial interaction language for supporting children in developing cultural understanding and awareness.

Currently the whole system is prepared for usage with Japanese children, to investigate whether the pictorial interaction language and the serious game are understood in an Asian culture as well. Therefore, the provided grammatical structure of the interface had to be slightly adapted. 
Acknowledgments. This work was funded by the European Commission within the 7th Framework Program under grant agreement eCute (education in cultural understanding, technologically enhanced).

\section{References}

1. Bowman, D.A.: 3D User Interfaces. In: The Encyclopedia of Human-Computer Interaction. The Interaction Design Foundation, Aarhus (2013)

2. Forlines, C., Wigdor, D., Shen, C., Balakrishnan, R.: Direct-touch vs. mouse input for tabletop displays. In: Proceedings of the SIGCHI Conference on Human Factors in Computing Systems, CHI 2007 (2007)

3. Nazir, A., Ritter, C., Aylett, R., Krumhuber, E., Swiderska, A., Degens, N., Endrass, B., Hume, C., Hodgson, J., Mascarenhas, S.: ECUTE: DIFFERENCE IS GOOD! In: IADIS International Conference on e-Learning, pp. 425-429 (2012)

4. Aylett, R., Lim, M.Y., Hall, L., Endrass, B., Tazzyman, S., Ritter, C., Nazir, A., Paiva, A., Hofstede, G.J., Andre, E., Kappas, A.: Werewolves, cheats, and cultural sensitivity. In: Proc. of 13th Int. Conf. on Autonomous Agents and Multiagent Systems, AAMAS 2014 (2014)

5. Plotkin, A.: Werewolf: A Mind Game (2010), http://www. eblong.com/zarf/werewolf.html

6. Sali, S., Wardrip-Fruin, N., Dow, S., Mateas, M., Kurniawan, S., Reed, A.A., Liu, R.: Playing with words: From intuition to evaluation of game dialogue interfaces. In: Proceedings of the Fifth International Conference on the Foundations of Digital Games, FDG 2010, pp. 179-186. ACM, New York (2010)

7. Aylett, R.S., Vala, M., Sequeira, P., Paiva, A.C.R.: Fearnot! - An emergent narrative approach to virtual dramas for anti-bullying education. In: Cavazza, M., Donikian, S. (eds.) ICVS-VirtStory 2007. LNCS, vol. 4871, pp. 202-205. Springer, Heidelberg (2007)

8. Bondy, A.S., Frost, L.A.: The picture exchange communication system. Focus on Autism and Other Developmental Disabilities 9(4) (1994)

9. Mirenda, P.: Toward functional augmentative and alternative communication for students with autism. Language, Speech, and Hearing Services in Schools 34, 203-216 (2003)

10. Symbols Worldwide Ltd T/A Widgit Software: Widgit, http://www.widgit.com/index.php (last viewed: April 2, 2014)

11. SymbolWorld, http://www.symbolworld.org/ (last viewed: April 2, 2014)

12. Antle, A.: The design of cbc4kids storybuilder. In: Interaction Design and Children (IDC 2003), pp. 59-68 (2003)

13. Kelleher, C., Pausch, R., Kiesler, S.: Storytelling Alice Motivates Middle School Girls to Learn Computer Programming. In: CHI 2007, pp. 1455-464. ACM (2007)

14. Takasaki, T., Mori, Y.: Design and Development of a Pictogram Communication System for Children Around the World. In: Ishida, T., R. Fussell, S., T. J. M. Vossen, P. (eds.) IWIC 2007. LNCS, vol. 4568, pp. 193-206. Springer, Heidelberg (2007)

15. Damian, I., Endrass, B., Huber, P., Bee, N., André, E.: Individualized Agent Interactions. In: Allbeck, J.M., Faloutsos, P. (eds.) MIG 2011. LNCS, vol. 7060, pp. 15-26. Springer, Heidelberg (2011)

16. Read, J.: Validating the Fun Toolkit: An instrument for measuring childrens opinions of technology. In: Cognition Technology and Work (2007) 